This item was submitted to Loughborough's Research Repository by the author.

Items in Figshare are protected by copyright, with all rights reserved, unless otherwise indicated.

\title{
Analysis of the crystallization process of a biopharmaceutical compound in the presence of impurities using process analytical technology (PAT) tools
}

PLEASE CITE THE PUBLISHED VERSION

http://dx.doi.org/10.1002/jctb.4743

\section{PUBLISHER}

(c) John Wiley \& Sons

VERSION

AM (Accepted Manuscript)

\section{PUBLISHER STATEMENT}

This work is made available according to the conditions of the Creative Commons Attribution-NonCommercialNoDerivatives 4.0 International (CC BY-NC-ND 4.0) licence. Full details of this licence are available at: https://creativecommons.org/licenses/by-nc-nd/4.0/

\section{LICENCE}

CC BY-NC-ND 4.0

\section{REPOSITORY RECORD}

Simone, Elena, Wei Zhang, and Zoltan K. Nagy. 2016. "Analysis of the Crystallization Process of a Biopharmaceutical Compound in the Presence of Impurities Using Process Analytical Technology (PAT) Tools". figshare. https://hdl.handle.net/2134/21451. 


\section{Analysis of the crystallization process of a}

\section{2 biopharmaceutical compound in the presence of}

\section{3 impurities using process analytical technology}

\section{$4 \quad$ (PAT) tools}

5 Elena Simone, ${ }^{1}$ Wei Zhang, ${ }^{2,3}$ Zoltan K. Nagy*1,3

6

$7 \quad{ }^{1}$ Department of Chemical Engineering, Loughborough University, Loughborough, LE11

8 3TU, U.K.

$9 \quad{ }^{2}$ New drug research and development center, North China Pharmaceutical group

10 Corporation, Shijiazhuang, China

$11{ }^{3}$ School of Chemical Engineering, Purdue University, West Lafayette, 47907, Indiana,

12 United State 


\section{Abstract}

2 The crystallization of biopharmaceuticals can be problematic since, because the biosynthesis

3 of these compounds is very difficult to control, they can present a significant amount of

4 impurities that has to be eliminated. In fact, impurities can lead to changes in the properties of

5 the drug that can significantly reduce its effectiveness or even put in danger the user.

6 The substance used in this work is vitamin B12 crude product extracted from fermentation.

7 The aim of this work is to exploit process analytical technology (PAT) tools to study the

8 crystallization step of vitamin B12. Linear cooling crystallization experiments were

9 performed using different conditions. The effects of solvent, cooling rate, seeding and purity

10 of the initial material on the final size distribution and purity of the crystals were investigated

11 through the use of UV/Vis spectroscopy, focused beam reflectance measurement (FBRM)

12 and the CryPRINS software (Crystallization Process Informatics System).

13 It was found that impurities strongly inhibit the growth of vitamin B12 crystals, promoting

14 nucleation and leading to a poor final crystal size distribution. Slow cooling can help in

15 increasing the purity of the final product but also generates a broad crystal size distribution

16 because of secondary nucleation. Preparing the solution with material already crystallized

17 once and using purified seeds helped in obtaining a narrower crystal size distribution and also

18 reduces breakage.

19

20 Keywords: PAT tools, biopharmaceutical, vitamin, crystallization, monitoring 


\section{Introduction}

2 Over the last decade the investment in research and development of biopharmaceutical

3 companies has more than doubled: there is a great interest in formulating and optimizing the

4 manufacturing of bio-molecules and biopharmaceuticals such as proteins, lipids, vitamins,

5 hormones and DNA. Those compounds can be used in the treatment of serious diseases or

6 just as nutritional supplement.

7 Vitamin B12 (cobalamin) is one of the biggest and most complex vitamins and, in humans, it

8 is required to assist the actions of two enzymes: methionine synthase and (R)-methylmalonyl-

9 CoA mutase. It is commonly used to cure pernicious anemia and its deficiency determines an

10 increase in homocysteine levels that leads to a major risk for heart disease, stroke,

11 atherosclerosis and vascular diseases. Despite the lack of solid evidences on the importance

12 of vitamin B12 in maintaining normal myelination of nerve cells, its role in preventing many

13 neurological and psychiatric symptoms is becoming clearer and clearer. For these reasons,

14 every year, around 10 tons of the semisynthetic version of vitamin B12 (cyanocobalamin) are

15 produced by biofermentation from several bacterial species. ${ }^{1}$

16 A schematic of vitamin B12 molecule is shown in Figure 1. The molecule is fairly large and 17 complex and can be divided in three parts: (1) a central corrin ring which contains four 18 ligands for the central cobalt ion, (2) a lower ligand donated by the 5,619 dimethylbenzimidazole (DMBI) and (3) an upper ligand made from either an adenosyl group 20 or a methyl group.

21 Two pseudo-polymorphic forms were discovered by X-ray diffraction: a wet and a dry 22 crystalline structure. ${ }^{2,3}$ The main difference between the two is the presence of water 23 molecules in the crystal lattice that makes the unit cell of the wet form bigger than the dry.

24 Cyanocobalamin is mainly produced by biosynthetic fermentation processes, although a full 25 chemical synthesis (consisting of 70 steps) was developed in the early 70 s. $^{1}$ High levels of 
1 impurities are present in the biosynthesized vitamin B12 that can inhibit growth of the 2 crystals and strongly affect the final crystal size distribution together with the ease of 3 downstream processes (filtration in particular).

4 Process analytical technology (PAT) tools are widely used for the development of the 5 crystallization processes of many synthetic pharmaceuticals. ${ }^{4-6}$. The term PAT refers to "a 6 system for designing, analyzing, and controlling manufacturing trough timely measurement 7 of critical quality and performance attributes of raw and in-process material and processes, 8 with the goal of ensuring final product quality". ${ }^{7}$ PAT tools are the main element used in the 9 “Quality by Design” (QbD) approach that was introduced in the pharmaceutical industries in 10 order to minimize product waste due to mistakes in the manufacturing process. The QbD 11 concept consists in obtaining the desired quality of the product though the correct design of the manufacturing process. In this approach some critical variables of the process, strictly related to the quality of the product, are controlled during the manufacturing in order to 14 control the quality of the product itself. ${ }^{8,9}$ The QbD approach is well known and applied in 15 the pharmaceutical industry but only few examples are present for the manufacturing of 16 biopharmaceuticals. ${ }^{10,11}$ Despite the difficulty of controlling biological processes Near infrared (NIR) and Raman spectroscopy, HPLC and image analysis were recently used for some manufacturing steps of biomolecules. ${ }^{12-14}$ In this work the crystallization of vitamin B12 will be studied with PAT tools (ATR-UV/Vis, FBRM and CryPRINS) and the effect of impurities on the growth and nucleation rate will be analyzed. The produced crystals are analyzed with optical microscopy, HPLC and Raman. Most of the impurities in cyanocobalamin have high levels of fluorescence and, therefore, Raman can give an indication of the purity of the crystals since it is highly sensitive to this

24 phenomenon. ${ }^{15,16}$ However, for a precise quantification of the type and level of impurity in 25 vitamin B12 samples HPLC is necessary. The paper provides one of the first comprehensive 
1 case studies that illustrate how an array of PAT tools can be used for the systematic

2 understanding and design of a biopharmaceutical crystallization process.

\section{$4 \quad$ Materials and methods}

\section{Equipment}

6 Crude Vitamin B12 produced by fermentation was donated by Hebei Welcome 7 Pharmaceutical Co., LTD (China). The raw material consisted in a dry powder containing 8 around 7\% of impurities. Those were mainly byproducts of the fermentation process (e.g. 509 carboxyl cyanocobalamin, 34-methyl cobalamin, 8-epi-cyanocobalamin and 10 cyanocobalaminic lactate) and inert. Ultrapure water was obtained via a Millipore ultra-pure 11 water system while ethanol was purchased by Fisher Scientific (purity $>95 \%$ v/v). Pure water or solutions of water and ethanol were used as solvents for the experiments.

13 Experiments were conducted at both Loughborough University, UK and Purdue University, 14 USA with similar, but not exactly identical instruments. Two $500 \mathrm{ml}$ glass jacketed vessels 15 and the same Raman system (an RXN1 Raman analyzer with immersion probe and $785 \mathrm{~nm}$ 16 laser, Kaiser with iC Raman 4.1 software) were used. A simple schematic of the rig used for 17 the experiments is shown in Figure 2. The experiments at Loughborough University were performed using an MSC621 Carl Zeiss ATR-UV/Vis spectrophotometer with Hellma ATR (type 661.822-UV) probe and a LabView based in-house software, a D600L Lasentec FBRM probe (Mettler Toledo with FBRM software V 6.7.0) and a Huber Ministat CC3

21 thermoregulator. The data from the FBRM, ATR-UV/Vis and the Huber is transmitted in real-time to the CryPRINS software (Crystallization Process Informatics System). This allows real-time monitoring and control of the FBRM counts, ATR-UV/Vis signal and the

24 temperature, as well as setting a temperature profile and performing different control strategies. The instrumentation at Purdue University consisted in a Zeiss MCS621 UV/Vis 
1 spectrophotometer with UV-VIS $190-720 \mathrm{~nm}$ Zeiss probe (Zeiss ProcessXplorer software

2 version 1,3-Build 1.3.1.30), an FBRM G400 0.5-2000microns (Mettler Toledo with ic.FBRM

3 software version 4.3) and a JULABO F25-ME thermoregulator. The samples of vitamin

4 produced were analyzed using a Raman RXN1 microprobe (Kaiser) and an HPLC Agilent

51100 series (Hewlett Packard) with ChemStation software rev. A.09.03[1417].

$6 \quad$ Procedure for linear cooling experiments

7 Mixtures of water and ethanol and pure water were used as solvents for the linear cooling

8 experiments. The concentrations used were about $0.118-0.13 \mathrm{~g} / \mathrm{g}$ solvent in water and ethanol

9 (saturation temperature of about $50{ }^{\circ} \mathrm{C}$ ) and between 0.09 and $0.11 \mathrm{~g} / \mathrm{g}$ solvent for water

10 (saturation temperature of about $60^{\circ} \mathrm{C}$ ). The material was dissolved and the hot solution was

11 filtered (using a filter paper) before starting the cooling profile because of the presence of insoluble particles in the raw material. The solubility of vitamin B12 in water at different temperatures was provided by the supplying company and is shown in Table 1, while data for ethanol/water mixtures at different temperatures was not available from the literature and the amount of material donated was too small to accurately measure it. However, two inferential curves of the solubility expressed as UV signal (absorbance value at $361 \mathrm{~nm}$ ) were obtained in order to compare two solvents. Those curves can also be directly compared with the UV data in the Results and Discussion section (which is actually an absorbance and not a concentration value). The curves were acquired by slow heating $\left(0.075{ }^{\circ} \mathrm{C} / \mathrm{min}\right)$ of two slurries of vitamin B12 in water and ethanol/water. The amount of vitamin dissolved at any time and temperature is proportional to the absorbance value recorded. Figure 3 shows the inferential curves as well as the exact solubility in the two solvents at high temperatures (120 $\mathrm{g} / \mathrm{L}$ ethanol/water solution at $53{ }^{\circ} \mathrm{C}$, and $84 \mathrm{~g} / \mathrm{L}$ water at $62{ }^{\circ} \mathrm{C}$ ). The material was added to the solvent at ambient temperature and then dissolved by heating the solution and keeping it at high temperature for 15-20 min. In ethanol and water the higher temperature was around 
$175{ }^{\circ} \mathrm{C}$ while in pure water it was around $70^{\circ} \mathrm{C}$. Four cooling rates were used: $-0.075,-0.1$, -

20.5 and $-1{ }^{\circ} \mathrm{C} / \mathrm{min}$ (slower, slow, fast and faster cooling), both seeded and unseeded

3 experiments were performed. The cooling rates were chosen based on experience with similar

4 types of crystals, equipment and scale. ${ }^{19-21}$ The final temperature after cooling was 5-6 ${ }^{\circ} \mathrm{C}$. In

5 the case of the seeded experiments the mass of seeds was of about $2.5 \%$ of the total solid

6 dissolved in the vessel. Seeds were obtained after three successive crystallizations of the raw

7 material. The same seeds were used for all the seeded experiments: crystals were in the form

8 of short needles with fairly homogenous width (around $40-60 \mu \mathrm{m}$ ) and variable length (up to

9 around $200 \mu \mathrm{m})$. At the end of the profile, the temperature was kept constant for few hours

10 (until both FBRM and ATR-UV/Vis signals stabilized); after that, crystals were filtered and

11 washed with acetone. CryPRINS was used to select the desired heating/cooling rate and to monitor the UV and FBRM signals during the experiments. In addition to the linear cooling experiments, three consecutive crystallizations of the same material were conducted using ethanol and water or pure water as solvents. Recrystallizing the same material more than once allows obtaining very pure crystals but the overall yield of the process is penalized. These three experiments were conducted to check the purity that could be obtained by using multiple steps of crystallization compared to a single linear cooling profile. Table 2 shows the conditions of the experiments performed in this section. HPLC analysis

The mobile phase for the HPLC analyses was prepared dissolving $7.4 \mathrm{~g}$ of $\mathrm{Na}_{2} \mathrm{HPO}_{4} \cdot 2 \mathrm{H}_{2} \mathrm{O}$ 21 (HPLC purity grade, Fisher Scientific) in $740 \mathrm{ml}$ of deionized water (from a Millipore Elix 5) and then adding $260 \mathrm{ml}$ of methanol (Fisher Scientific). The solution $\mathrm{pH}$ was adjusted to 3.5 using $\mathrm{H}_{3} \mathrm{PO}_{4}$ (Fisons analytical reagents). The solid samples were dissolved in purified water

24 (concentration of about $1 \mathrm{mg} / \mathrm{ml}$ ). For the detection of the sample constituents the UV wavelength at $351 \mathrm{~nm}$ was used. The flow rate applied was $0.8 \mathrm{ml} / \mathrm{min}$ for a total running 
1 time of 40 min for each sample. The temperature of the column was kept constant at $35{ }^{\circ} \mathrm{C}$

2 and the injection size was $20 \mu$ l. The type of column used was an Analytical Column Waters

3 Spherisorb ${ }^{\circledR} 5 \mu \mathrm{m}$ C8 $4.6 \times 250 \mathrm{~mm}$. The main peak at about $14 \mathrm{~min}$ corresponds to

4 cyanocobalamin while the other peaks are byproducts (50-carboxyl cyanocobalamin, 34-

5 mehil cobalamin, 8-Epi- cyanocobalamin, 7 $\beta, 8 \beta$-cyanocobalamin lactate) and other

6 unspecified impurities. All samples were analyzed at least twice and purity was calculated as

7 the average relative area of the cyanocobalamin peak.

8 Principal component analysis on Raman spectroscopic data

9 Principal components analysis (PCA) is a dimension reduction technique for quantitative 10 data. Given a set of data in more variables, with PCA it is possible to reduce the dimensions 11 of the problem down to two or three, keeping at the same time, enough information to capture the inherent variability within the data. PCA is a method that models all the variations in the data set using orthogonal basis vectors (eigenvectors) which are called principal components

14 in this analysis. ${ }^{17}$ If $A$ is the $n \times m$ matrix of input data ( $n$ rows of spectra recorded at $m$ 15 wavelengths for example), PCA can decompose it in the following linear system:

$16 \quad A=C P^{T}+\varepsilon$,

17 where $C$ is the $n \times k$ matrix of $k$ principal components' scores and $P$ is the $m \times k$ matrix

18 of the eigenvectors of $A$. The eigenvectors are called "loadings" in PCA. $\varepsilon$ is a matrix 19 containing the unexplained variance. Loadings and score for PCA can be used to interpret the 20 data. The scores are usually represented in two-dimensional scatter plots. Often, a very high percentage of variability is gathered in the first few principal components so only a few of

22 these plots are necessary. The scores plots are usually used to detect similarities, differences 23 or other interesting relationships among samples. A PCA was performed over Raman 24 spectroscopic data of vitamin B12 in order to determine a relation between the purity of the 
1 analyzed crystals and their Raman spectra. Matlab 2013a was used for the calculation of the

2 principal component scores (pca function).

\section{$4 \quad$ Results and discussion}

\section{$5 \quad$ Linear cooling experiments}

6 Simple linear cooling was applied to the raw and recrystallized vitamin B12 and the results

7 were studied using FBRM and images from an optical microscope. The crystal size

8 distribution (CSD) was found to be strongly affected by the purity of the material used and

9 the cooling rate. Usually large crystals and a narrow crystal size distribution are preferred

10 because they facilitate the downstream operations (such as filtration, washing and milling).

11 For this reason a good quality CSD is characterized by large crystals of similar size. The solvent used did not have a significant effect on the CSD but it affects the final purity of the crystals. The effect of seeding was also analyzed and it was found that a slightly purer material can be obtained at the end of the crystallization using purified seed. Figure 4 shows the results of experiment 1 and experiment 2 described in Table 2, in which raw vitamin B12 was crystallized from an ethanol/water mixture at two different cooling rates ( -0.5 and $0.1{ }^{\circ} \mathrm{C} / \mathrm{min}$, fast and slow cooling). From the microscopic images of crystals at the end of the two experiments (see Figure 4a and Figure 4b) it can be noticed that in both cases the size distributions look very broad with both small and large crystals; but a considerably larger size is clearly generated by the slow cooling shown in Figure 4b. This behaviour is similar to what normally happens for common pharmaceuticals such as paracetamol. ${ }^{18-21}$ In general, during a fast cooling experiment, nucleation happens at high superaturation generating a large amount of small nuclei while in a slow cooling experiment, few nuclei are nucleated at low supersation. However, for paracetamol, a fast cooling generates a high number of total counts from the FBRM due to the large number of nucleai generated at high supersaturation. In the 
1 case of Vitamin B12 the trend seems to be the opposite, as shown in Figure 5a and b. In 2 experiment 1 (Figure 5a) when slow cooling was used, the number of counts was lower than 3 in experiment 2 (Figure 5b) which was conduced at a fast cooling rate. Furthermore, the 4 images from the microscope seem to contrast with the data from FBRM data since, clearly 5 less and bigger particles were generated using slow cooling (Figure 4b) compared to fast 6 cooling (Figure 4a). Additionally, the final square weighted mean chord length distribution is 7 higher for the fast cooling rate (Figure 5a) than the slow one (Figure 5b). This discrepancy 8 between images and FBRM data is related to the needle shape of the vitamin B12 crystals. As 9 the particles grow in length, the shorter size of the crystals is counted by the FBRM more 10 times than the longer giving the erroneous larger number of counts for small crystal size 11 present in the vessel. A similar behavior for this probe was observed by Leyssens et al. for a small needle-like molecule prodrug antagonist called CDP323-2. ${ }^{22}$

13 The longer the crystals are, the higher is the value of the total counts recorded by FBRM; and 14 that is the reason why slow cooling of raw vitamin B12 generated a higher value of the total 15 counts than fast cooling (70,600 \#/meas. in the slow cooling against 44,000 \#/meas. as shown 16 in Figure 4a and 4b). However, considering the large amount of small crystals present at the end of both experiments (in particular see Figure 4a for experiment 1 and Figure 4c for experiment 3), it is clear that secondary nucleation prevails on growth for raw vitamin B12. This can be explained by the fact that the presence of impurities inhibits growth, and supersaturation is used mainly to nucleate new small crystals.

21 Some breakage is also present in the slow cooling after $400 \mathrm{~min}$ as shown in the CLD 22 distribution of Figure 5d, where a reduction in the number of larger particles can be observed. 23 Breakage of crystals also contributed to the higher number of counts in the case of slow 24 cooling. Figure 6a and 6c show the FBRM and UV data for fast and slow cooling 25 crystallization of a purified solution of vitamin B12 (experiment 3 and 4 of Table 2). The use 
1 of recrystallized material with fewer impurities reduced considerably both primary and

2 secondary nucleation during the cooling phase and promoted growth of the existing crystals.

3 Figure $6 \mathrm{~b}$ and $6 \mathrm{~d}$ show the microscopic images of the resulting crystals; the crystal size

4 distribution is narrower compared to experiment 1 and 2 and crystals are considerably larger

5 with the maximum total counts that reaches only 5,200 \#/meas. in the fast cooling experiment

6 and 26,900\#/meas. for the slow cooling. The effect of the shape of the crystals on the FBRM

7 data is clearer in these two experiments because of the absence of breakage or secondary

8 nucleation; crystals in Figure 6d are clearly longer than the ones shown in Figure 6b and the

9 CLD (Figure 7a and 7b) present a bimodal shape with a growing peak at low sizes due to the

10 increasing counts of the shorter size of the crystals.

11 It is clear that growth of vitamin B12 is strongly inhibited by impurities and very broad crystal size distributions are generated by secondary nucleation and breakage. Additionally, in some cases, multiple nucleation events can happen as shown in Figure 8b, c and d, which

14 is also a clear indication of growth inhibited crystallization processes in the presence of impurities. Figure 8a shows the results for experiment 5 (Table 2) where vitamin B12 nucleated and grew throughout the entire cooling period. Figure 8b, 8c and 8d instead, show cases in which a second major nucleation event happened during the cooling phase. The UV signal dropped suddenly during the second nucleation and the counts increased at the same time. This phenomenon appeared with both raw and recrystallized material, for seeded and unseeded experiments and for both the solvents used. During these experiments growth was so inhibited that supersaturation accumulated during the cooling until it reached a level at which a second major nucleation event could happen. In these cases the final CLD is very broad and many small particles are present as a result of this double nucleation as shown in Figure 9 (for experiment 7). 


\section{Purity analysis using HPLC and Raman spectroscopy}

2 A first qualitative screening of both the raw material and the crystals obtained by consecutive

3 crystallizations (with increasing purity as shown in Table 3) was performed using Raman

4 microscopy. The inert material filtered during the crystallizations was also analyzed. Figure

5 10a shows the Raman spectra of raw and filtered material; a strong fluorescence, due to

6 impurities can be noticed (high intensity and no defined peaks). It is interesting to observe

7 that just the filtration of the hot solution eliminates part of the fluorescent material. A loss of

8 fluorescence due to less impurity can also be noticed in the samples of the consecutive

9 crystallizations (Figure 10b). The number and position of the peaks is the same for all the

10 samples but they tend to become broader and less intense as fluorescence (and, therefore

11 impurity) increases. In order to have some quantitative information from Raman spectroscopy

12 a principal component analysis was performed over the Raman spectra of the recrystallized

13 material, the raw material and the filtered one. Figure 11a shows the score of principal

14 component PC2 plotted versus the score of principal component PC1. The raw and filtered

15 material points are isolated and very far from the crystallized samples because of their high

16 impurity content. Figure 11b shows only the crystallized samples: a clear trend of the scores

17 as a function of purity is present. This example provides an illustration for the nonconventional use of Raman spectroscopy for monitoring low concentration of impurities based on fluoresce. While fluorescence is in general undesirable in Raman spectroscopy,

20 since it makes the interpretation of the significant peaks difficult, monitoring the amount of

21 fluorescence in the Raman spectra can serve as a method of detecting the existence and changes in impurity levels in the case of fluorescent impurities.

23 The loss of impurity during the consecutive crystallizations also considerably improved the

24 size of the crystals as shown in Figure 12. The raw material showed in Figure 12a is highly 25 fluorescent and amorphous; particles are very small and hygroscopic and the colour is intense 
1 red. Crystalline vitamin B12 is darker as shown in Figure 12b to $12 \mathrm{~d}$. The mean size of the

2 crystals increases during the consecutive crystallization as a result of less inhibited growth.

3 The results of HPLC analyses of the samples from selected experiments out of those

4 performed are shown in Table 3. A higher purity can be reached if slow cooling rates are

5 applied and seeding also helps in increasing the purification efficiency. The largest increase

6 in purity was reached using raw material in an ethanol/water mixture with seeding and slow

7 cooling (linear cooling experiment 14, increase in purity of 3.65\%). The second

8 crystallizations (using purified material) as expected present a lower increase in purity;

9 however, even for this set of experiments, slow cooling and seeding helped reaching a higher

10 purity. The effect of the solvent on the final crystal size distribution is negligible but choosing

11 the suitable solvent seems to help increasing the purity of the product. It also seems not to

12 affect purification efficiency since the final purity of similar experiments in different solvents

13 is very close.

15 Conclusions

16 A set of PAT tools was used to study the crystallization in the presence of impurities of a 17 small biomolecule produced by fermentation (vitamin B12). Materials produced by 18 biosynthesis often contain a high amount of impurities that can significantly reduce the 19 quality of the final crystals in term of size distribution. Multiple successive recrystallizations 20 of the same material can help eliminating impurities and reaching a better crystal size 21 distribution, but they also contribute to decrease the yield. Using PAT tools can help understanding the characteristics of the studied compound and developing a correct crystallization strategy to improve crystal size and purity of the final product.

24 It was found that impurities strongly affect the growth rate, promoting nucleation and, in 25 some cases, generating multiple nucleation events during the cooling profile. The final crystal 
1 size distribution for these cases was found to be very broad, with very large crystals together

2 with significant amounts of fines. Slow cooling of very impure material increases the

3 probability of multiple nucleation events and, therefore, leads to a broad crystal size

4 distribution. However, slow cooling was found to generate more pure crystals compared to

5 fast cooling. The best quality of the crystals, in terms of size, was reached using already

6 crystallized material to prepare the solution and slow cooling rates. Seeding was also found to

7 help in increasing the purity of the final crystal while a change in the solvent composition did

8 not largely affect purity or final CSD. The needle shape of the vitamin B12 crystals also had

9 to be considered before interpreting the FBRM results. The growth of the long size of the

10 needles generates an increase in the FBRM total counts that can be confused with nucleation.

11 An analysis of the crystals with the optical microscope was necessary to interpret the counts

12 and chord length distribution recorded by the FBRM. Monitoring the level of fluorescence

13 due to impurities in the Raman spectrum, can serve as a method of detecting changes in the

14 impurity concentrations in the crystalized products.

15

16 Acknowledgments

17 Financial support provided by the European Research Council grant no. [280106-CrySys].

18 The authors are grateful to Hebei Welcome Pharmaceutical Co., LTD (China), for donating

19 vitamin B12. 


\section{References}

2 1. Martens, J.H., Barg, H., Warren, M.J., Jahn, D., Microbial production of vitamin B12. Appl Microbiol Biotechnol 58: 275-285 (2002).

2. Hodgkin, D.C., Kamper, J., Mackay, M., Pickworth, J., Structure of vitamin B12. Nature 5 178: 64-66 (1956).

3. Hodgkin, D.C., Pickworth, J., Robertson, J.H., Trueblood, K.N., Prosen, R.J., White, J.G., Structure of vitamin B12. Nature 4477: 325-328 (1955).

4. Nagy, Z.K., Braatz.,R., Advances and new directions in crystallization control. Annu. Rev Chem Biomol Eng, 3: 55-75 (2012).

5. Nagy, Z.K., Fevotte, G., Kramer, H., Simon, L.L., Recent advances in the monitoring, modelling and control of crystallization systems. Chem Eng Res Des 91(10): 1903-1922 (2013).

6. Simon, L.L., Pataki, H., Marosi, G., Meemken, F., Hungerbühler, K., Baiker, A., Tummala, S., Glennon, B., Kuentz, M., Steele, G., Kramer, H.J.M., Rydzak, J.W., Chen, Z., Morris, J., Kjell , F., Singh, R., Gani, R., Gernaey, K.V., Louhi-Kultanen, M., O’Reilly, J., Sandler, N., Antikainen, J., Yliruusi, J., Frohberg, P., Ulrich, J., Braatz, R.D., Leyssens, T., von Stosch, M., Oliveira, R., Tan, R.B.H., Wu, H., Khan, M., O’Grady, D., Pandey, A., Westra, R., Delle-Case, E., Pape, D., Angelosante, D., Maret, Y., Steiger, O., Lenner, M., Abbou-Oucherif, K., Nagy, Z.K., Litster, J. D., Krishna Kamaraju, V., Chiu, M.S., Assessment of Recent Process Analytical Technology (PAT) Trends: A Multiauthor Review. Org Process Res Dev 19(1): 3-62 (2015). 
1 7. Department of Health and Human Services, Food and Drug administration, PAT Guidance for Industry: A Framework for Innovative Pharmaceutical Development, Manufacturing and Quality Assurance. Rockville, MD (2004).

8. Rathore, A.S., Bhambure, R., Ghare, V., Process analytical technology (PAT) for biopharmaceutical products. Anal Bioanal Chem 398: 137-154 (2010).

9. Rathore, A.S., Winkle, H., Quality by design for biopharmaceuticals. Nature Biotechnol 27(1): 26-34 (2009).

10. Read, E.K., Shah, R.B., Riley, B.S., Park, J.T., Brorson, K.A., Rathore, A.S., Process Analytical Technology (PAT) for biopharmaceutical products: Part II. Concepts and applications. Biotechnol Bioengi 105(2): 285-295 (2010).

11. Glassey, J., Gernaey, K.V., Clemens, C., Schulz, T.W., Oliveira, R., Striedner, G., Mandenius, C.F., Process analytical technology (PAT) for biopharmaceuticals. Biotechnol J 6: 369-377 (2011).

12. Bhambure, R., Kumar, K., Rathore, A.S. 2011. High-throughput process development for biopharmaceutical drug substances. Trends Biotechnol 29(3): 127-135.

13. Smith, D., Process monitoring and control using live cell imaging for the manufacturing of cell therapies. Doctoral thesis, Loughborough University (2014).

14. Ündey, C., Ertunç, S., Mistretta, T., Looze, B., Applied advanced process analytics in biopharmaceutical manufacturing: challenges and prospects in real-time monitoring and control. J Process Control 20: 1009-1018 (2010). 
1 15. Mayer, E., Gardiner, D.J., Hester, R.E., Resonance Raman spectra of vitamin B12 and dicyanocobalamin. Biochim Biophys Acta 297: 568-570 (1973).

16. Zhang, Z., Wang, B., Yin, Y., Mo, Y., Surface-enhanced Raman spectroscopy of vitamin B12 on silver particles in colloid and in atmosphere. J Mol Struct 927: 88-90. (2009)

18. Abu Bakar, M.R., Nagy, Z.K., Saleemi, A.N., Rielly, C.D., The impact of direct nucleation control on crystal size distribution in pharmaceutical crystallization processes, Cryst Growth Des, 9:1378-1384 (2009).

19. Saleemi, A.N., Rielly, C.D., Nagy, Z.K. 2012a, Automated direct nucleation control for in situ dynamic fines removal in batch cooling crystallization, CrystEngComm, 14: 2196-

22. Leyssens, T., Baudry, C., Hernandez, M.L.E., Optimization of a crystallization by online FBRM analysis of needle-shaped crystals. Org Process Res Dev, 15: 413-426 (2011). 
1 Table 1: Solubility of vitamin B12 in water at different temperature (data provided by Hebei Welcome 2 Pharmaceutical Co., LTD)

\begin{tabular}{cc}
\hline Temperature $\left({ }^{\circ} \mathbf{C}\right)$ & Solubility (g/l water) \\
\hline 10 & 9.25 \\
\hline 20 & 10 \\
\hline 25 & 11.5 \\
\hline 30 & 13.5 \\
\hline 45 & 24 \\
\hline
\end{tabular}

3

4

5 


\begin{tabular}{|c|c|c|c|c|c|}
\hline Experiment & Solvent & $\begin{array}{c}\text { Vitamin } \\
\text { concentration } \\
\text { (g/g solvent) }\end{array}$ & Type of material & Cooling rate $\left({ }^{\circ} \mathrm{C} / \mathrm{min}\right)$ & Seeding \\
\hline 1 & Ethanol/water & 0.118 & Raw & -0.5 (fast cooling) & No \\
\hline 2 & Ethanol/water & 0.118 & Raw & -0.1 (slow cooling) & No \\
\hline 3 & Water & 0.097 & $\begin{array}{c}\text { Crystallized } \\
\text { (more than once) }\end{array}$ & -0.5 (fast cooling) & No \\
\hline 4 & Water & 0.097 & $\begin{array}{c}\text { Crystallized } \\
\text { (more than once) }\end{array}$ & -0.1 (slow cooling) & No \\
\hline 5 & Water & 0.089 & Raw material & -0.1 (slow cooling) & No \\
\hline 6 & Water & 0.106 & Crystallized & -0.075 (slower cooling) & No \\
\hline 7 & Water & 0.089 & Raw material & -0.1 (slow cooling) & Yes \\
\hline 8 & Water & 0.097 & Crystallized & -0.1 (slow cooling) & Yes \\
\hline 9 & Ethanol/water & 0.119 & Raw & -1 (faster cooling) & No \\
\hline 10 & Ethanol/water & 0.119 & Raw & -1 (faster cooling) & Yes \\
\hline 11 & Ethanol/water & 0.119 & Raw & -0.5 (fast cooling) & No \\
\hline 12 & Ethanol/water & 0.119 & Raw & -0.5 (fast cooling) & Yes \\
\hline 13 & Ethanol/water & 0.119 & Raw & -0.1 (slow cooling) & No \\
\hline 14 & Ethanol/water & 0.119 & Raw & -0.1 (slow cooling) & Yes \\
\hline 15 & Ethanol/water & 0.13 & Crystallized & -1 (faster cooling) & No \\
\hline 16 & Ethanol/water & 0.13 & Crystallized & -0.5 (fast cooling) & No(150 rpm) \\
\hline 17 & Ethanol/water & 0.13 & Crystallized & -0.5 (fast cooling) & No(240rpm) \\
\hline 18 & Ethanol/water & 0.13 & Crystallized & -0.5 (fast cooling) & Yes \\
\hline 19 & Ethanol/water & 0.13 & Crystallized & -0.1 (slow cooling) & No \\
\hline 20 & Ethanol/water & 0.115 & Raw & -0.5 & No \\
\hline 21 & Ethanol/water & 0.137 & From exp. 20 & -0.5 & No \\
\hline 22 & Water & 0.082 & From exp. 21 & -0.5 & No \\
\hline
\end{tabular}


Table 3: HPLC results for selected experiments performed

\begin{tabular}{|c|c|c|c|c|c|c|}
\hline Sample & $\begin{array}{c}\text { Purity } \\
\text { (\%) }\end{array}$ & $\begin{array}{c}\text { Improvement in } \\
\text { purity (\%) }\end{array}$ & Solvent used & $\begin{array}{c}\text { Material } \\
\text { used }\end{array}$ & $\begin{array}{c}\text { Cooling } \\
\text { rate } \\
\left({ }^{\circ} \mathrm{C} / \mathrm{min}\right)\end{array}$ & Seeded \\
\hline Raw material & 93.11 & 0 & n.a. & n.a. & n.a. & n.a. \\
\hline First crystallization & 96.14 & 3.03 & n.a. & n.a. & n.a. & n.a. \\
\hline $\begin{array}{c}\text { Second } \\
\text { crystallization }\end{array}$ & 97.29 & 1.16 & n.a. & n.a. & n.a. & n.a. \\
\hline $\begin{array}{c}\text { Third } \\
\text { crystallization }\end{array}$ & 98.03 & 0.74 & n.a. & n.a. & n.a. & n.a. \\
\hline LC experiment 8 & 97.54 & 1.24 & Water & Crystallized & -0.1 & Yes \\
\hline LC experiment 9 & 96.35 & 3.24 & Ethanol/water & Raw & -1 & No \\
\hline LC experiment 10 & 96.28 & 3.17 & Ethanol/water & Raw & -1 & Yes \\
\hline LC experiment 11 & 96.28 & 3.17 & Ethanol/water & Raw & -0.5 & No \\
\hline LC experiment 12 & 96.72 & 3.61 & Ethanol/water & Raw & -0.5 & Yes \\
\hline LC experiment 13 & 96.51 & 3.40 & Ethanol/water & Raw & -0.1 & No \\
\hline LC experiment 14 & 96.76 & 3.65 & Ethanol/water & Raw & -0.1 & Yes \\
\hline LC experiment 15 & 97.66 & 0.94 & Ethanol/water & Crystallized & -1 & No \\
\hline LC experiment 16 & 97.70 & 0.98 & Ethanol/water & Crystallized & -0.5 & No \\
\hline LC experiment 17 & 97.72 & 1.00 & Ethanol/water & Crystallized & -0.5 & No \\
\hline LC experiment 18 & 97.95 & 1.23 & Ethanol/water & Crystallized & -0.5 & Yes \\
\hline LC experiment 19 & 97.77 & 1.05 & Ethanol/water & Crystallized & -0.1 & No \\
\hline LC experiment 20 & 96.14 & 3.03 & Ethanol/Water & Raw & -0.5 & No \\
\hline LC experiment 21 & 97.29 & 1.16 & Ethanol/Water & $\begin{array}{c}\text { Crystallized } \\
\text { once }\end{array}$ & -0.5 & No \\
\hline LC experiment 22 & 98.03 & 0.74 & Water & $\begin{array}{c}\text { Crystallized } \\
\text { twice }\end{array}$ & -0.5 & No \\
\hline
\end{tabular}




\section{List of Figures:}

2 Figure 1: Molecular structure of cyanocobalamin. The CN group constitutes the difference

3 from the natural vitamin B12 and it is the results of the extraction procedure by which the

4 compound is removed from bacterial cultures. ${ }^{1}$

$5 \quad$ Figure 2: schematic of the equipment used during the experiments.

6 Figure 3: inferential solubility curve expressed as UV signal versus temperature in the two

7 solvents used. The addition of ethanol increased the solubility of vitamin B12 especially at

8 high temperature. This type of curve can replace the classical concentration versus

9 temperature relationship in cases in which only a small amount of material is available and

10 literature data are not available.

11 Figure 4: Microscope image of the crystals at the end of experiment 1 (fast cooling) (b)

12 Microscope image of the bigger crystals at the end of experiment 2 (slow cooling) (c)

13 Microscope image of the smaller crystals at the end of the experiment 2.

14 Figure 5: (a) Experiment 1: fast cooling of raw material in water and ethanol, $-0.5^{\circ} \mathrm{C} / \mathrm{min}$.

15 The graph shows the total counts per measurement and the mean of the square weighted

16 chord length distribution (MSWCLD) from the FBRM (b) Experiment 2: slow cooling of raw

17 material in water and ethanol, $-0.1^{\circ} \mathrm{C} / \mathrm{min}$. The graph shows the total counts per measurement and the mean of the square weighted chord length distribution from the FBRM (c)

19 Experiment 1: chord length distribution for the fast cooling experiment (d) Experiment 2:

20 chord length distribution for the slow cooling experiment.

21 Figure 6: Absorbance value at 361nm, Total counts from FBRM and mean of the square 22 weighted chord length distribution (MSWCLD) for (a) Experiment 3: fast cooling and 23 crystallization of purified material dissolved in water (b) Experiment 3: microscopic image of 24 the final crystals for the fast cooling experiment in water (c) Experiment 4: slow cooling and 
1 crystallization of purified material dissolved in water (d) Experiment 4: microscopic image of

2 the final crystals for the slow cooling experiment in water.

3 Figure 7: (a) Experiment 3: chord length distribution (CLD) during the fast cooling 4 experiment in water with purified material (b) Experiment 4: chord length distribution (CLD)

5 during the slow cooling experiment in water with purified material.

6 Figure 8: (a) Experiment 5: slow cooling $-0.1^{\circ} \mathrm{C} / \mathrm{min}$ of raw material. Total counts per 7 measurement from FBRM and absorbance from ATR-UV/Vis. (b) Experiment 6: slow 8 cooling $-0.075^{\circ} \mathrm{C} / \mathrm{min}$ of recrystallized material. Total counts per second from FBRM 9 (Loughborough University) and UV derivative signal. (c) Experiment 7: seeded and slow 10 cooling of raw material in water $\left(-0.1^{\circ} \mathrm{C} / \mathrm{min}\right)(\mathrm{d})$ Experiment 8: seeded and slow cooling of 11 recrystallized material in water $\left(-0.1^{\circ} \mathrm{C} / \mathrm{min}\right)$.

12 Figure 9: (a) Chord length distribution (CLD) in the seeded and slow cooling of raw material in water (Experiment 7) (b) Microscopic image of the final crystals for the seeded and slow 14 cooling of raw material in water (Experiment 7).

15 Figure 10: (a) Raman spectra of the raw material donated and the particles filtered at high 16 temperature (b) Raman spectra of the crystals from the three consecutive crystallizations. A 17 loss in fluorescence can be noticed.

18 Figure 11: (a) Score of the second principal component (PC2) plotted against the first principal component (PC1) for all the samples analysed. (b) Score of the second principal 20 component (PC2) plotted against the first principal component (PC1) for the crystallized 21 samples.

22 Figure 12: microscopic images of (a) Raw vitamin B12 from fermentation (b) Crystal 23 obtained after the first crystallization (c) Crystal at the end of the second crystallization (d) 24 Crystals after the third crystallization 


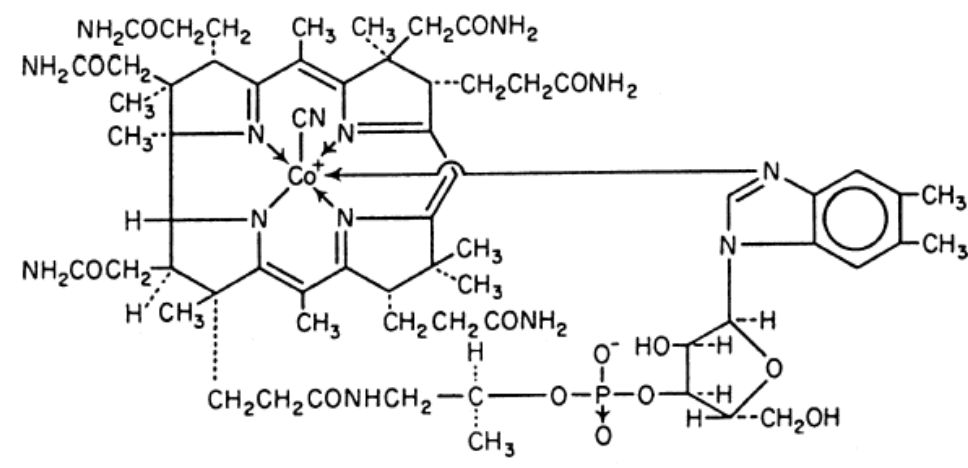

3 Figure 1: Molecular structure of cyanocobalamin. The $\mathrm{CN}$ group constitutes the difference from the natural vitamin $4 \quad$ B12 and it is the results of the extraction procedure by which the compound is removed from bacterial cultures. ${ }^{1}$ 


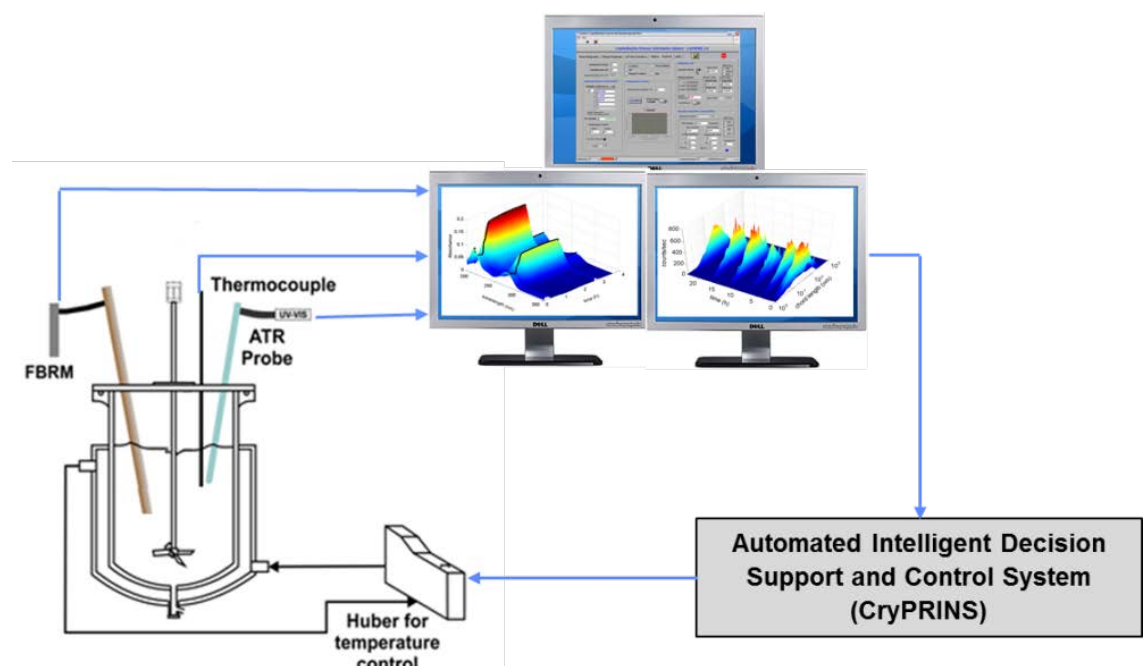

1

2 Figure 2: schematic of the equipment used during the experiments.

3 


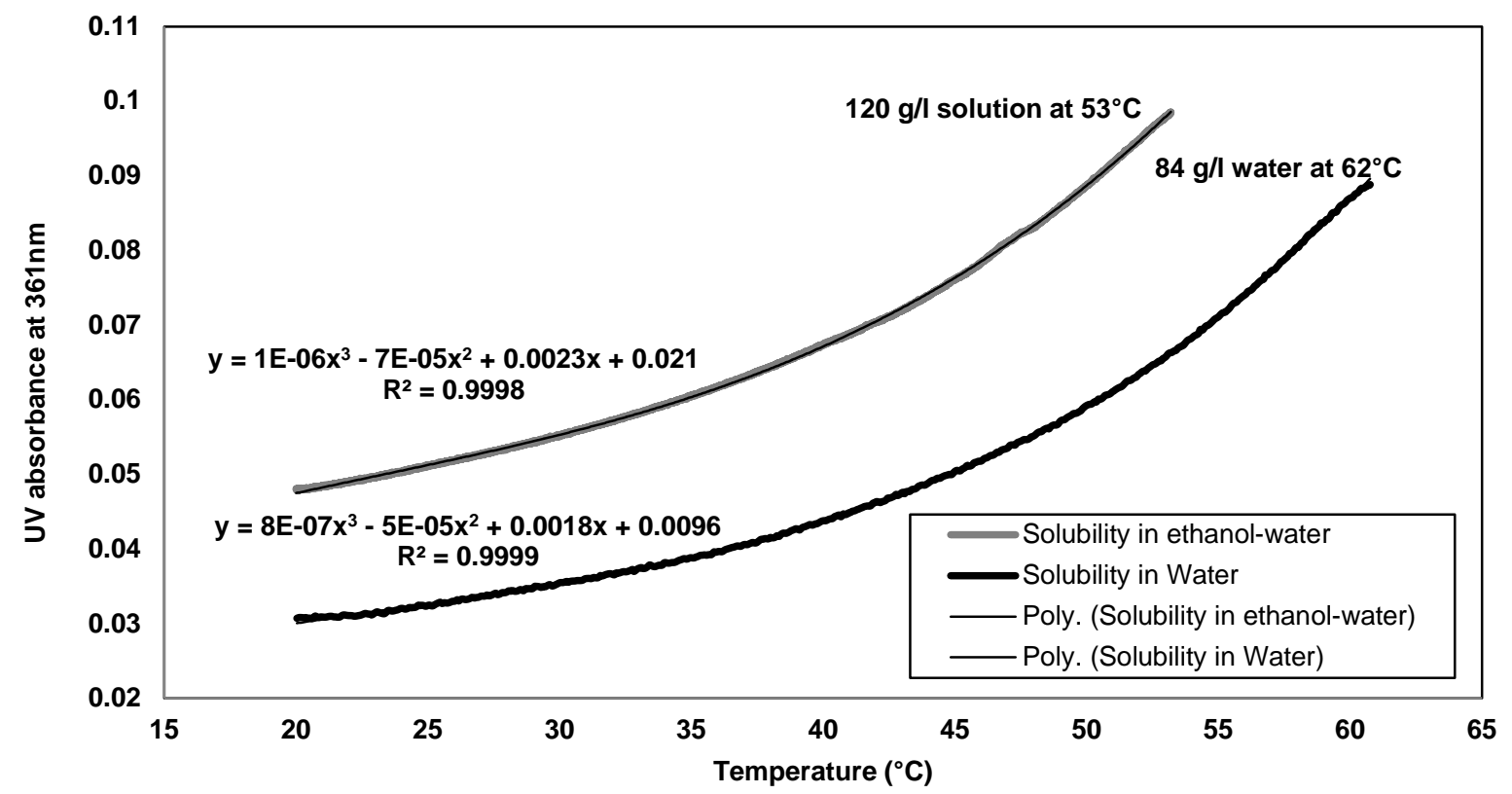

2 Figure 3: inferential solubility curve expressed as UV signal versus temperature in the two solvents used. The addition of ethanol increased the solubility of vitamin B12 especially at high temperature. This type of curve can replace the classical concentration versus temperature relationship in cases in which only a small amount of material is available and literature data are not available. 


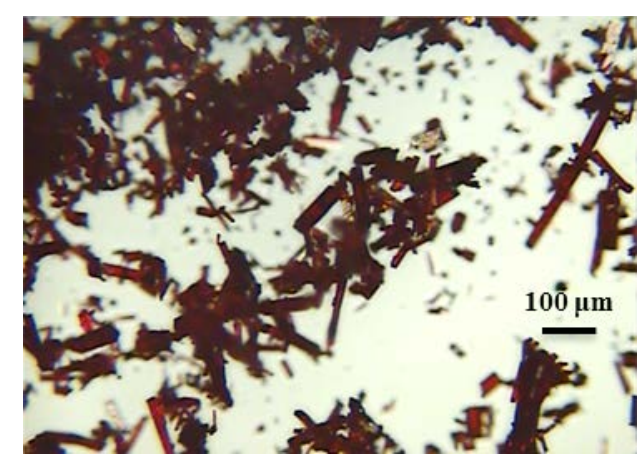

(a)

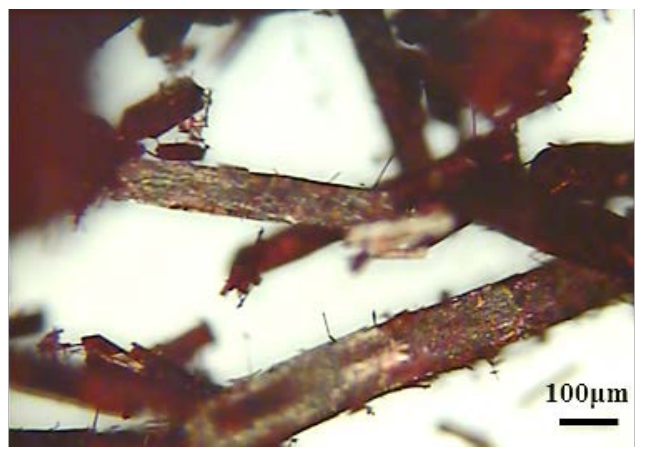

(b)

3

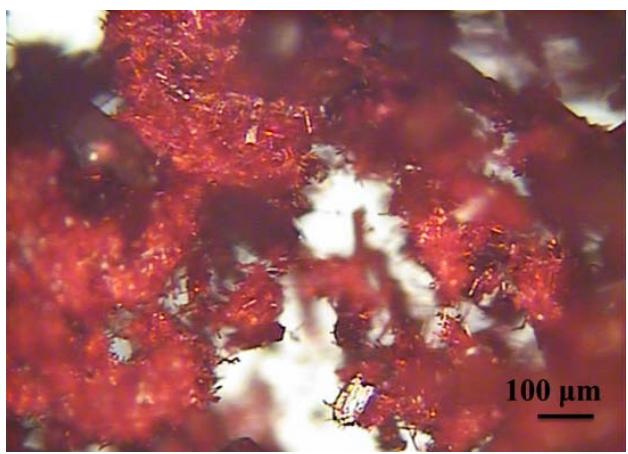

(c)

6

Figure 4: (a) Microscope image of the crystals at the end of experiment 1 (fast cooling) (b) Microscope image of the bigger crystals at the end of experiment 2 (slow cooling) (c) Microscope image of the smaller crystals at the end of the experiment 2. 


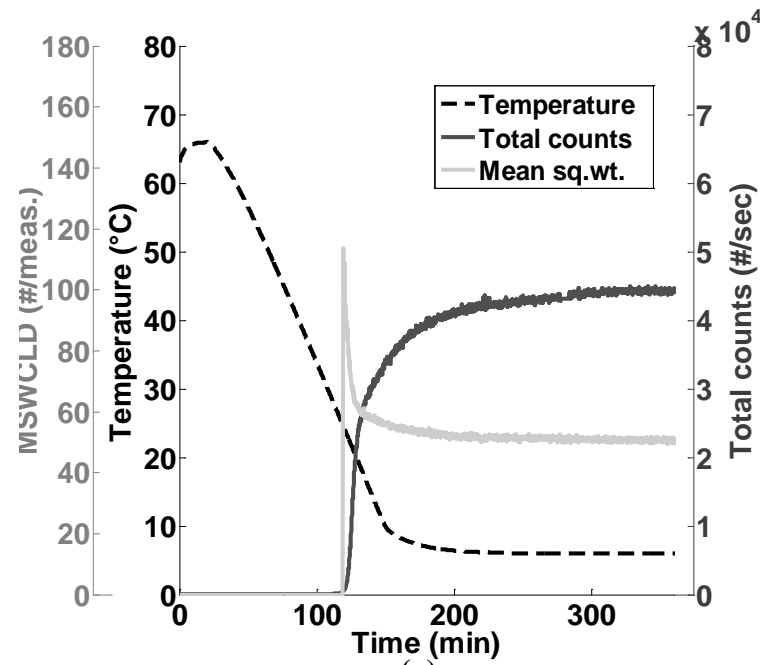

(a)

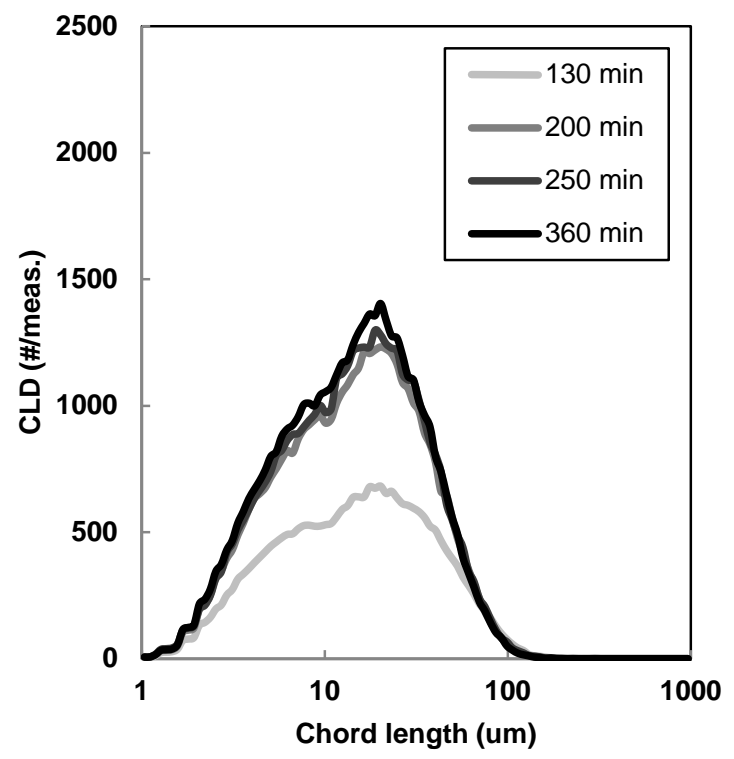

(c)

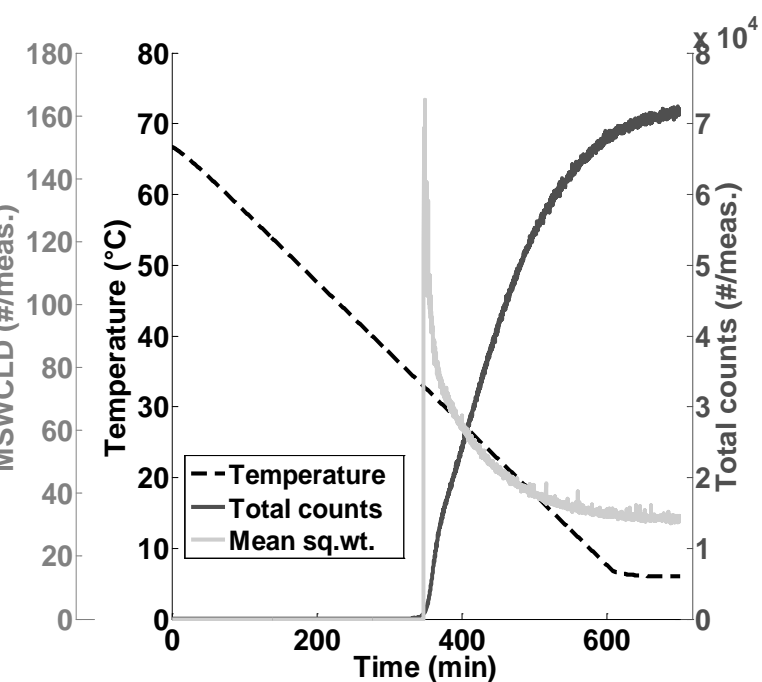

(b)

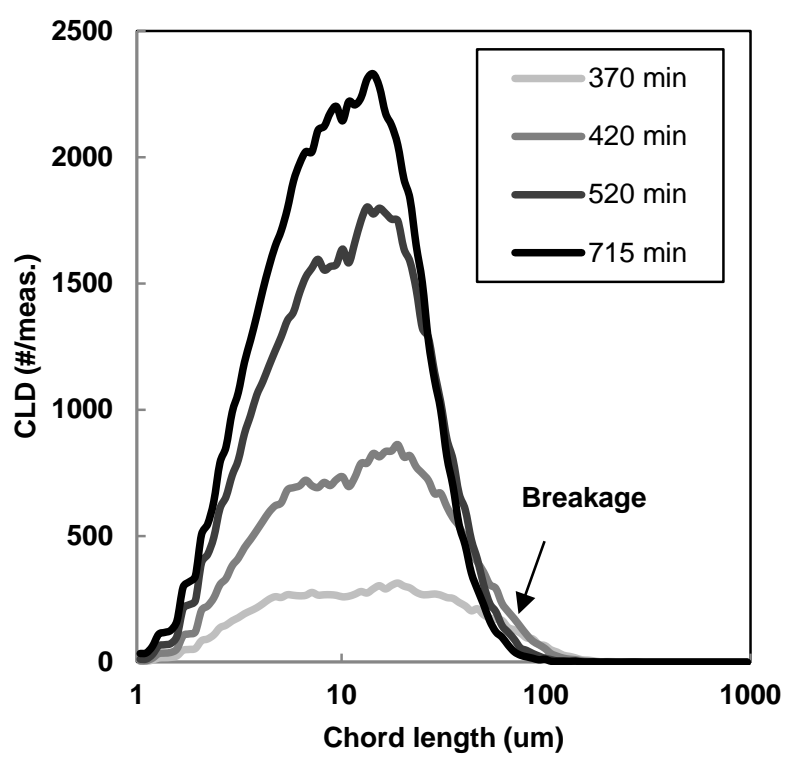

(d)

Figure 5: (a) Experiment 1: fast cooling of raw material in water and ethanol, $-0.5^{\circ} \mathrm{C} / \mathrm{min}$. The graph shows the total counts per measurement and the mean of the square weighted chord length distribution (MSWCLD) from the FBRM (b) Experiment 2: slow cooling of raw material in water and ethanol, $-0.1^{\circ} \mathrm{C} / \mathrm{min}$. The graph shows the total counts per measurement and the mean of the square weighted chord length distribution (MSWCLD) from the FBRM (c) Experiment 1: chord length distribution (CLD) for the fast cooling experiment (d) Experiment 2: chord length distribution (CLD) for the slow cooling experiment. 

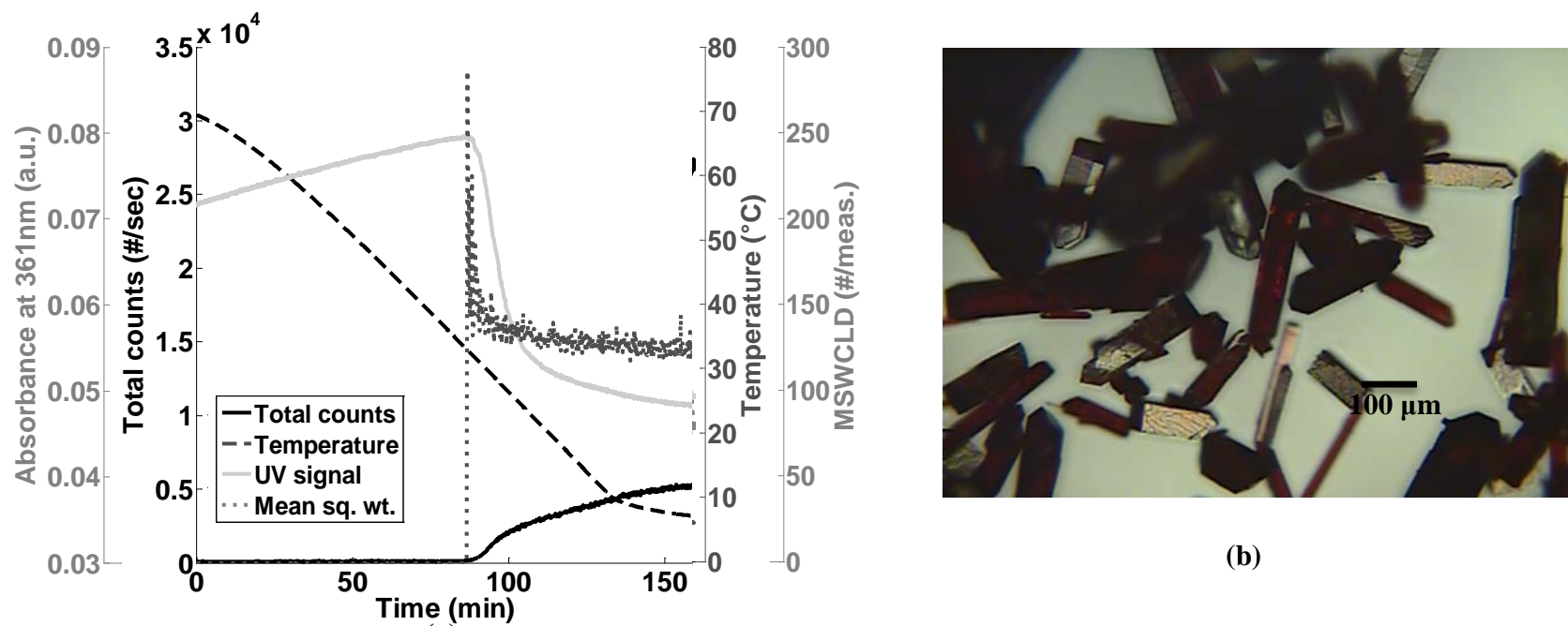

(b)

(a)
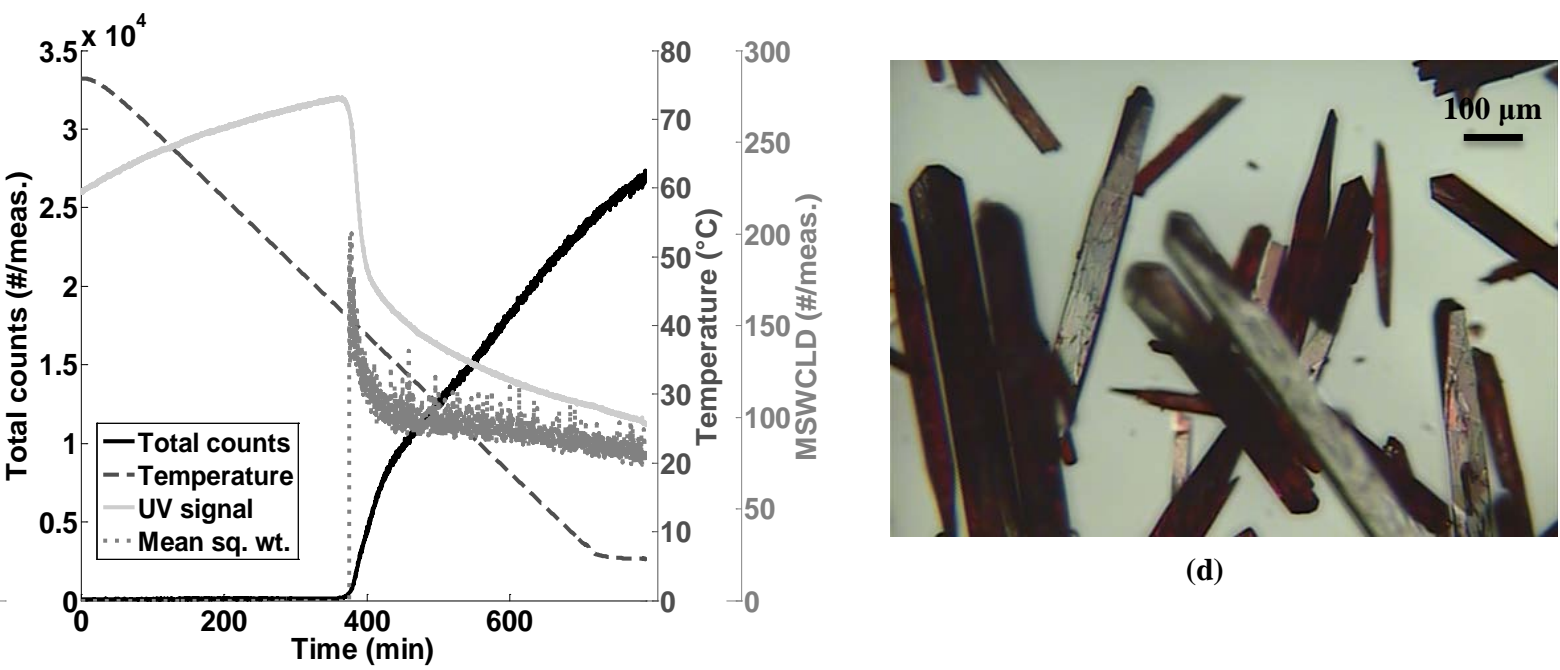

(d)

(c)

Figure 6: Absorbance value at $361 \mathrm{~nm}$, Total counts from FBRM and mean of the square weighted chord length distribution (MSWCLD) for (a) Experiment 3: fast cooling and crystallization of purified material dissolved in water (b) Experiment 3: microscopic image of the final crystals for the fast cooling experiment in water (c) Experiment 4: slow cooling and crystallization of purified material dissolved in water (d) Experiment 4: microscopic image of the final crystals for the slow cooling experiment in water. 


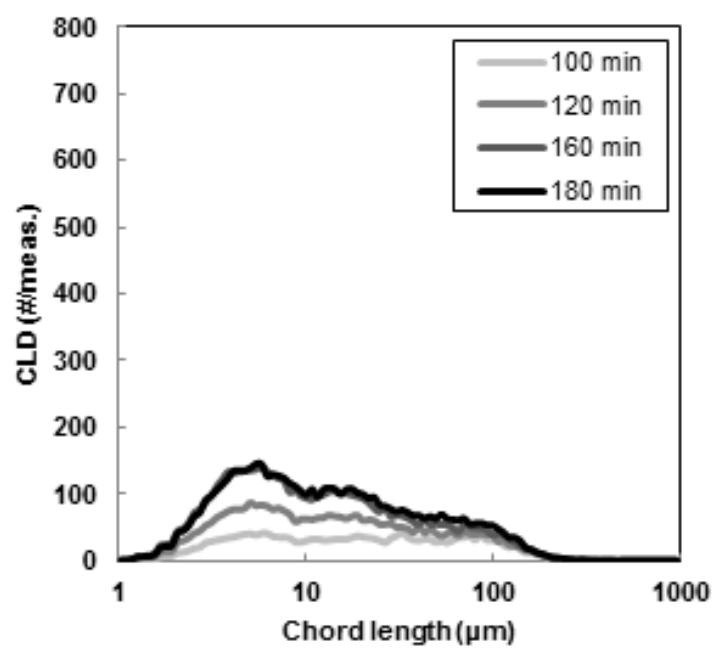

(a)

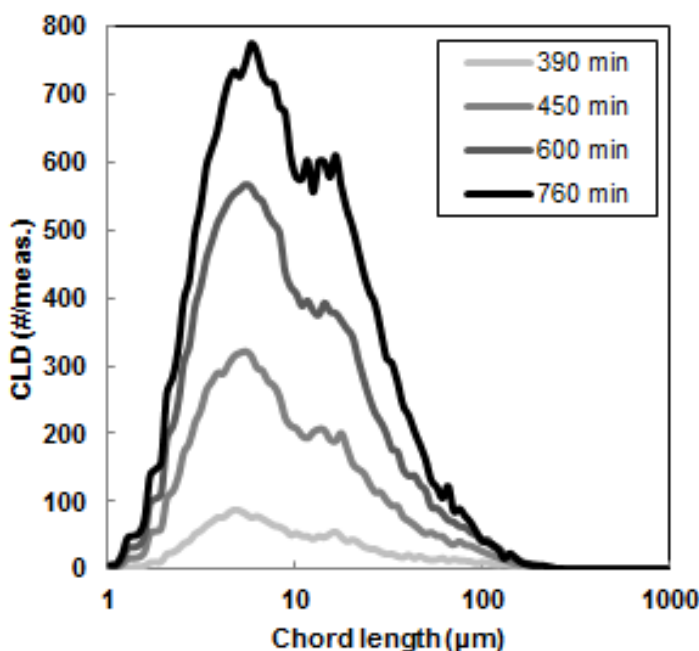

(b)

2 Figure 7: (a) Experiment 3: chord length distribution (CLD) during the fast cooling experiment in water with 3 purified material (b) Experiment 4: chord length distribution (CLD) during the slow cooling experiment in water 4 with purified material.

5 


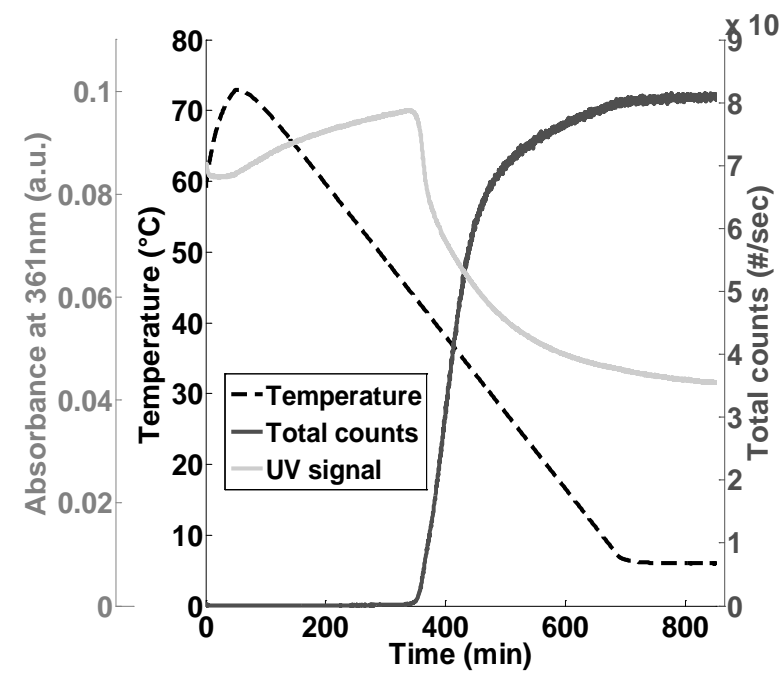

(a)

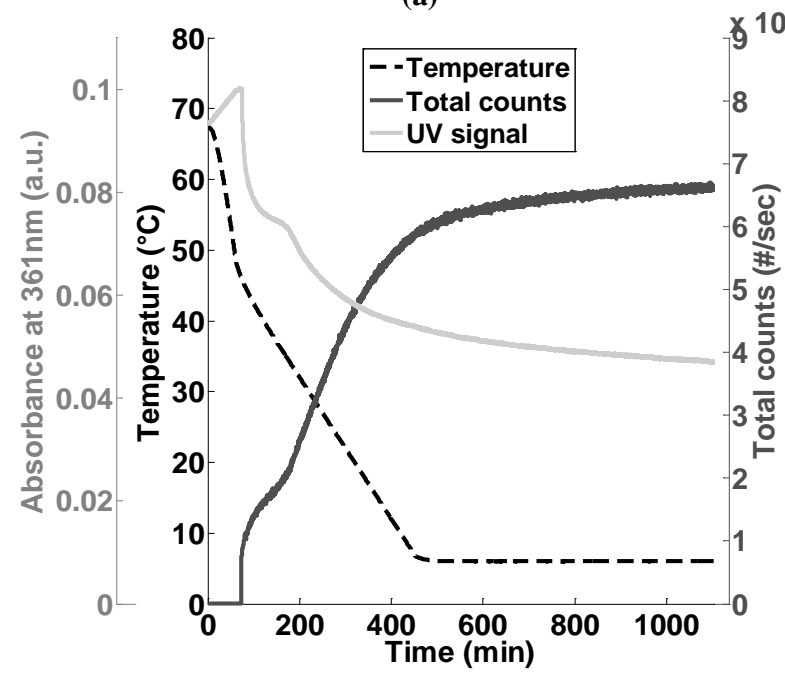

(c)

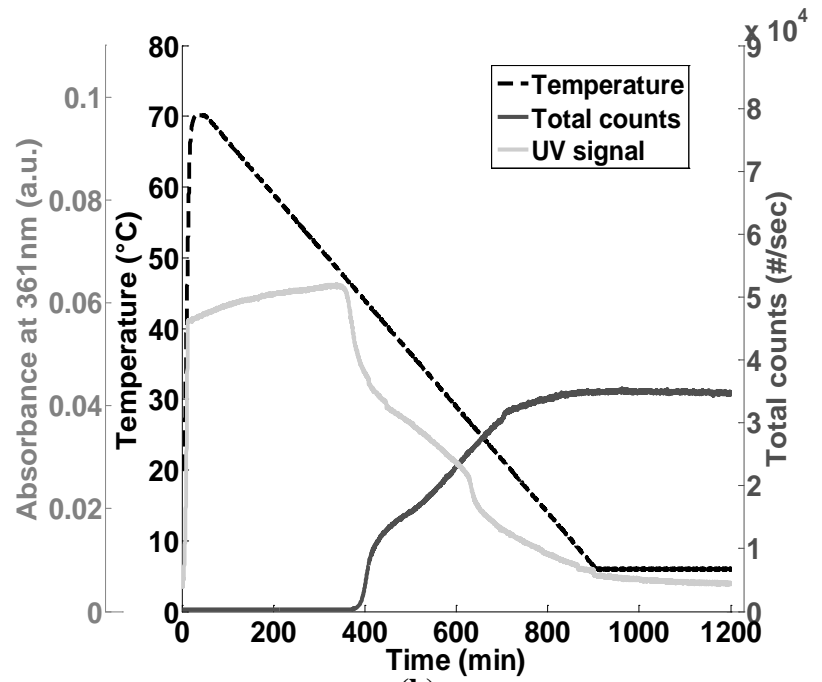

(b)
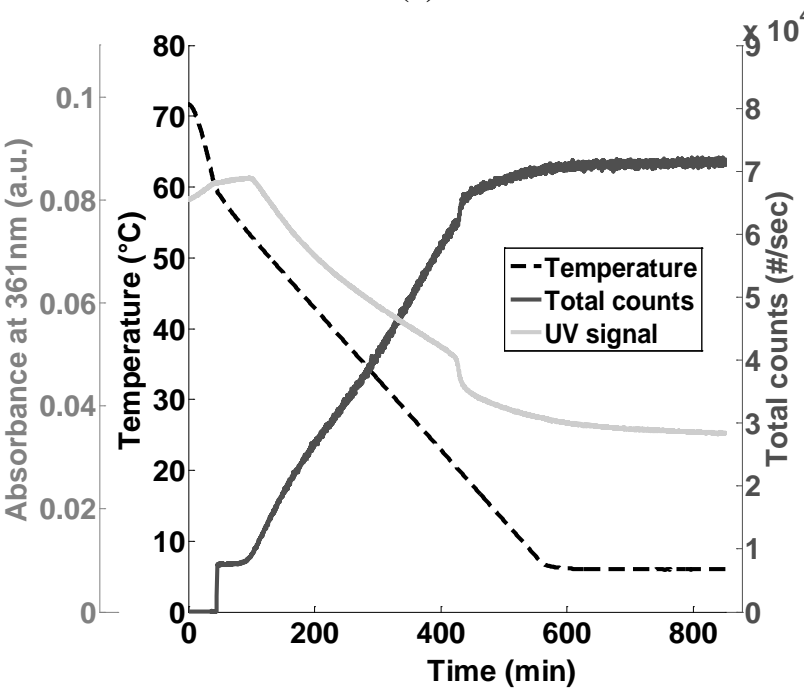

(d)

Figure 8: (a) Experiment 5: slow cooling $-0.1^{\circ} \mathrm{C} / \mathrm{min}$ of raw material. Total counts per measurement from FBRM and absorbance from ATR-UV/Vis. (b) Experiment 6: slow cooling $-0.075^{\circ} \mathrm{C} / \mathrm{min}$ of recrystallized material. Total counts per second from FBRM (Loughborough University) and UV absorbance. (c) Experiment 7: seeded and slow cooling of raw material in water $\left(-0.1^{\circ} \mathrm{C} / \mathrm{min}\right)$ (d) Experiment 8: seeded and slow cooling of recrystallized material in water ($\left.0.1^{\circ} \mathrm{C} / \mathrm{min}\right)$. Total counts per measurement from FBRM and absorbance from ATR-UV/Vis. 


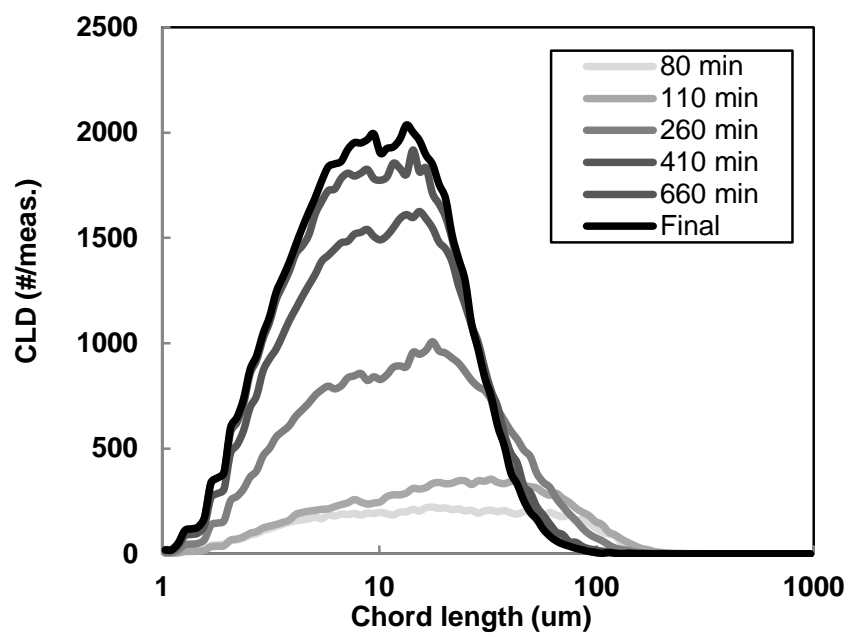

(a)

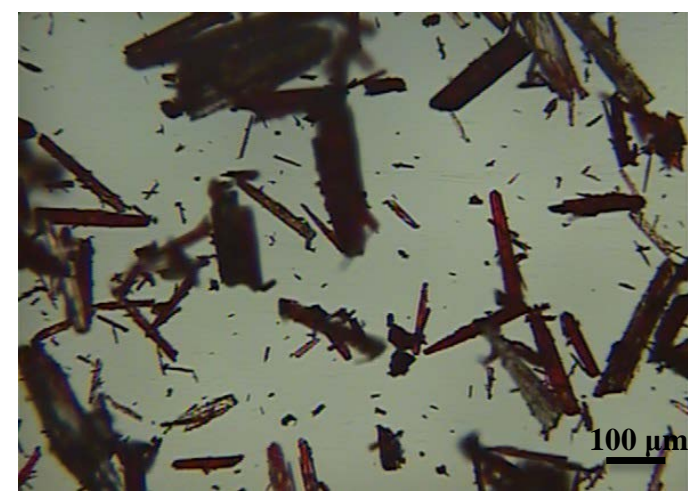

(b)

Figure 9: (a) Chord length distribution (CLD) in the seeded and slow cooling of raw material in water (Experiment 7) (b) Microscopic image of the final crystals for the seeded and slow cooling of raw material in water (Experiment 7). 


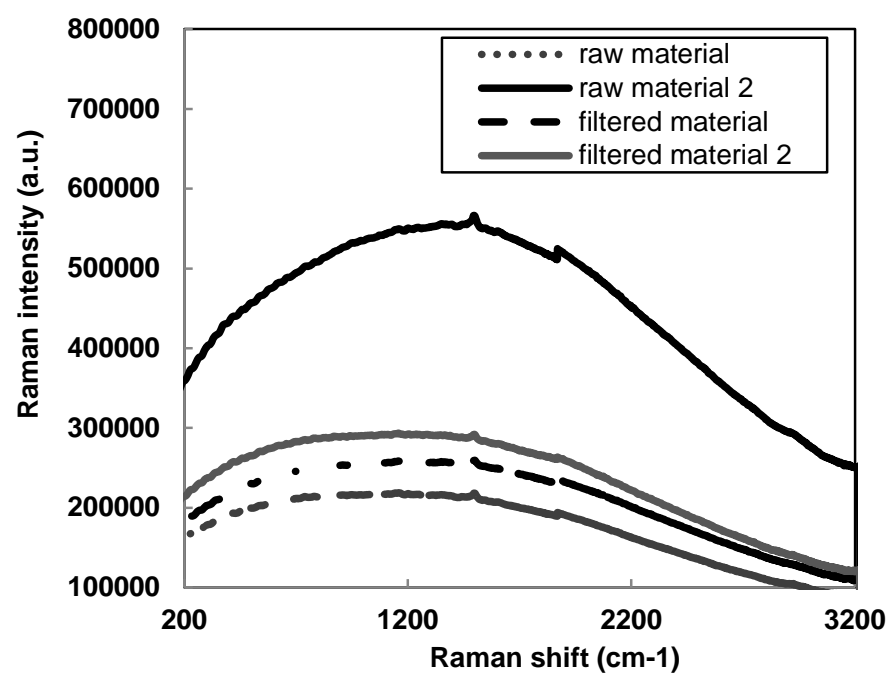

(a)

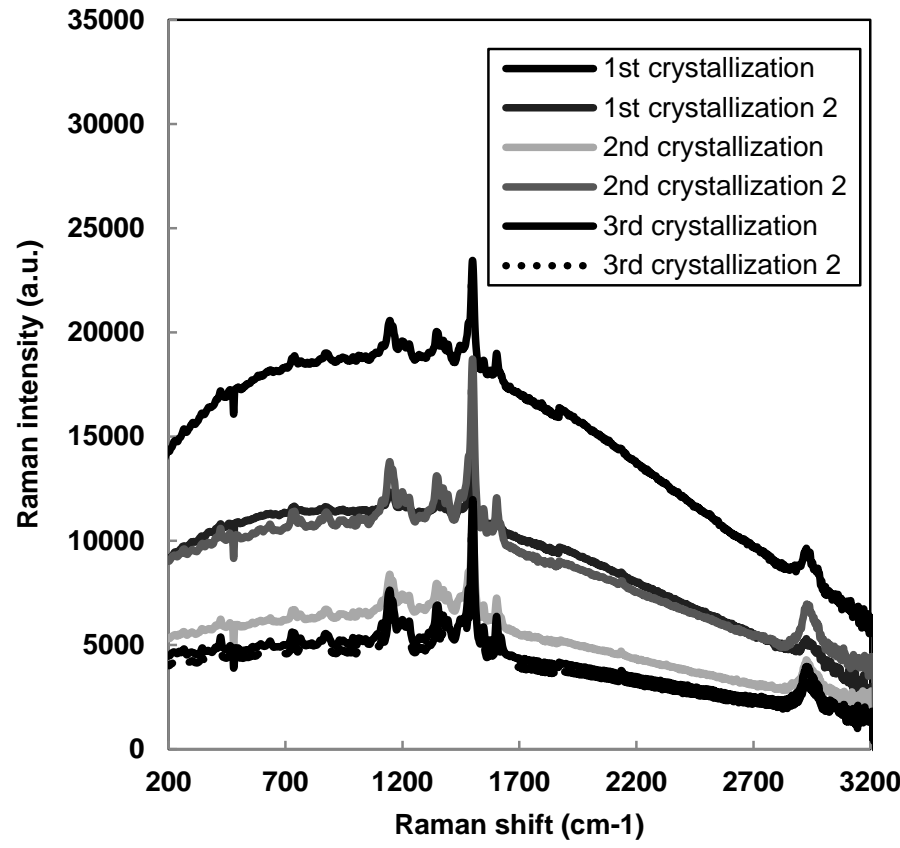

(b)

4 Figure 10: (a) Raman spectra of the raw material donated and the particles filtered at high temperature (b) Raman 5 spectra of the crystals from the three consecutive crystallizations. A loss in fluorescence can be noticed. 


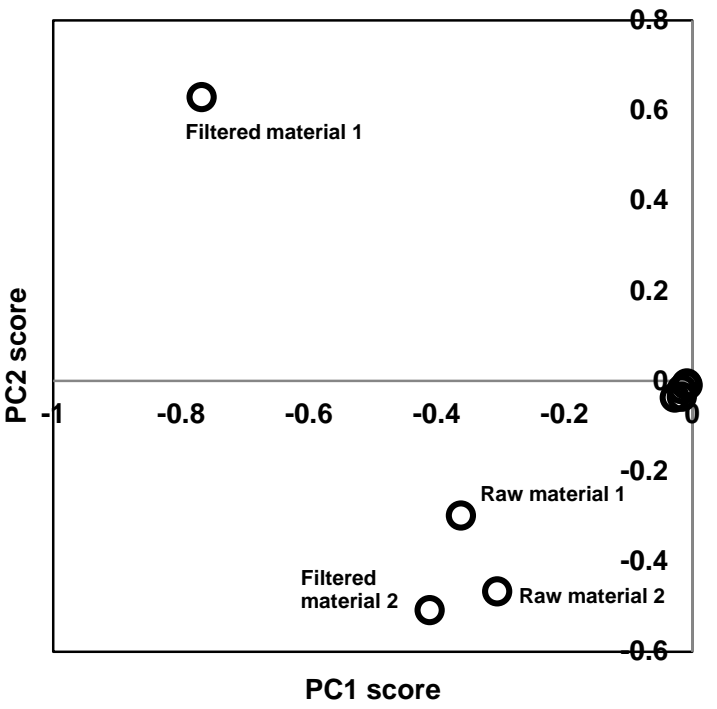

1

(a)

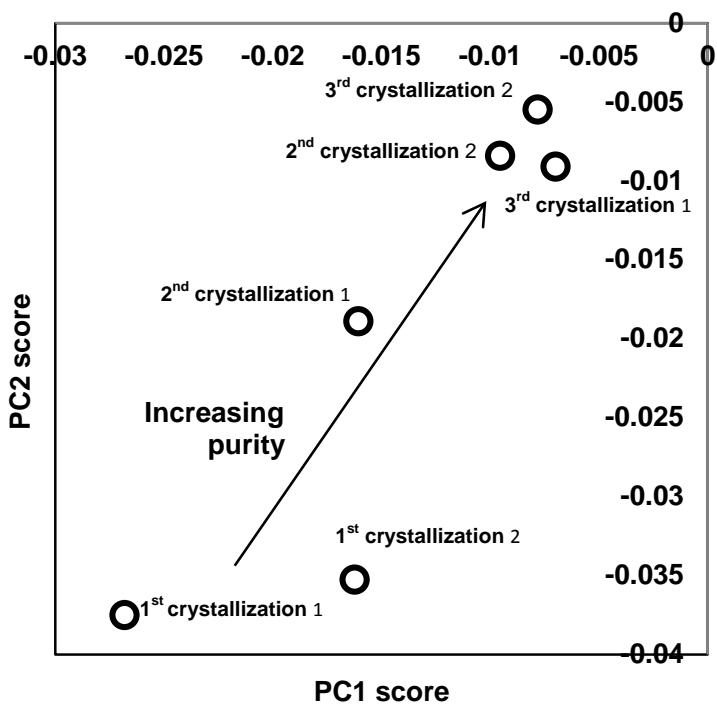

(b)

3 Figure 11: (a) Score of the second principal component (PC2) plotted against the first principal component (PC1) for 4 all the samples analysed. (b) Score of the second principal component (PC2) plotted against the first principal component (PC1) for the crystallized samples. 


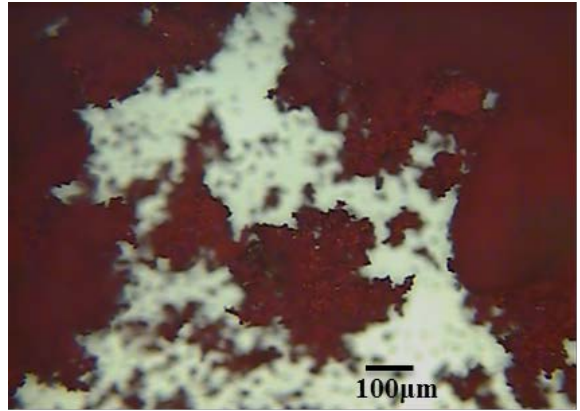

(a)

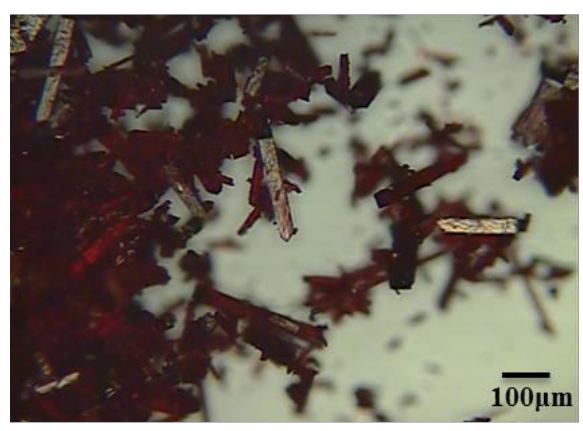

(c)

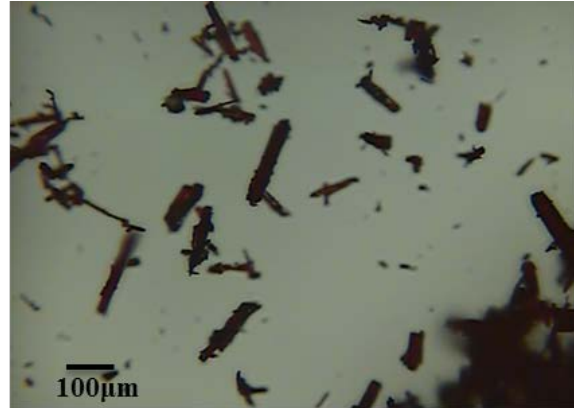

(b)

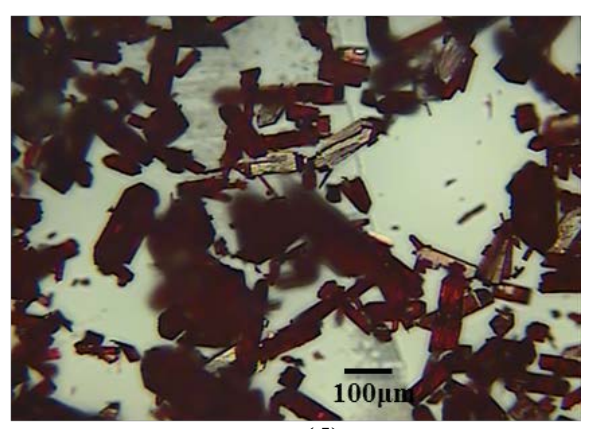

(d)

3 Figure 12: microscopic images of (a) Raw vitamin B12 from fermentation (b) Crystal obtained after the first 4 crystallization (c) Crystal at the end of the second crystallization (d) Crystals after the third crystallization 